\title{
Cystic fibrosis and survival to 40 years: a case-control study
}

\author{
N.J. Simmonds*, S.J. MacNeill ${ }^{\#}$, P. Cullinan ${ }^{\#}$ and M.E. Hodson*
}

ABSTRACT: The clinical course of patients with cystic fibrosis (CF) is variable and probably determined by many interacting factors. We aimed to examine the influence of early social and clinical factors on long-term survival.

A case-control study of adult CF patients was used to compare long-term survivors (aged $\geqslant 40 \mathrm{yrs}$ ) with patients who died before reaching 30 yrs of age. Each case $(n=78)$ was matched by birth date with at least one control $(n=152)$, after exclusion of "late diagnosis" patients. Probability-weighted logistic regression models were used to identify influences on survival.

Factors resulting in increased probabilities of survival included high body mass index (OR 1.76, 95\% Cl 1.40-2.22), forced expiratory volume in $1 \mathrm{~s}$ (OR per $5 \%$ increase $1.54,95 \% \mathrm{Cl} 1.32-1.80$ ), and forced vital capacity (OR per $5 \%$ increase $1.54,95 \% \mathrm{Cl} 1.33-1.78$ ) at transfer to the adult clinic and the exclusive use of oral antibiotics (OR $8.31,95 \% \mathrm{Cl} 3.02-22.88$ ). Factors resulting in decreased probabilities of survival were Pseudomonas aeruginosa acquisition (OR $0.18,95 \%$ 0.05-0.65) or pneumothorax before transfer to the adult clinic (OR $0.02,95 \% \mathrm{Cl} 0.004-0.08)$ and referral from a paediatric clinic in a deprived area (OR $0.13,95 \% \mathrm{Cl} 0.04-0.38$ ).

Long-term survival is associated with the clinical features present by the time of referral to an adult clinic. Even "early-diagnosis" disease appears to have different phenotypes, possibly independent of CF gene function, that have different survival patterns.

KEYWORDS: Adults, ageing, cystic fibrosis, survival

$\mathbf{T}$ he life expectancy of patients with cystic fibrosis (CF) has been steadily increasing despite the lack of a cure for the underlying cellular defect. Patients born today are expected to have a median survival into their 6 th decade [1]. The improvement has been explained in several ways including through the introduction of pancreatic enzymes, better nutrition, specialist-centre care, improved physiotherapy and more intensive antimicrobial treatment [2-4].

CF covers a wide spectrum of disease, from milder phenotypes with "non-classic" disease (with pancreatic sufficiency, milder lung disease and a later diagnosis), to more severe cases with a "classic" phenotype [5]. However, even within different groups there is variation in the rate of disease progression; some patients with features of classic disease run a mild course and indeed an important proportion of patients with the common "severe" $\Delta$ F508 mutation survive beyond $40 \mathrm{yrs}$ of age with relatively well-maintained lung function and weight $[6,7]$.

Thus, it has been hypothesised that other factors influence survival in CF. These include variations in the function of the responsible gene, the cystic fibrosis transmembrane conductance regulator (CFTR), and other independent genetic factors ("modifier" genes). None, however, has yet been shown directly to influence survival [8]. Other potential, nongenetic determinants of survival are so-called environmental influences; these cover a diverse range of factors, broadly divided into biological effectors (e.g. microorganisms, nutrition, sex and pollutants), social and cultural influences (e.g. socioeconomic status and adherence to treatment) and healthcare-related factors, such as access to care and interclinic treatment variations [9]. Evidence for or against these factors is variable and when they are most influential, or when an individual is most vulnerable to them, is not well understood. In view of this, we conducted a case-control study of long-term survival among patients registered with a specialist adult CF clinic with the aim of identifying early potential influences of longterm survival in patients diagnosed with $\mathrm{CF}$ in childhood.

\section{METHODS}

\section{Subjects}

Since 1965, details of all patients referred to the adult unit at Royal Brompton Hospital

\section{AFFILIATIONS}

*Depts of Cystic Fibrosis, and \#Occupational and Environmental Medicine, National Heart and Lung Institute, Royal Brompton Hospital and Imperial College London, London, UK.

CORRESPONDENCE N.J. Simmonds Dept of Cystic Fibrosis Royal Brompton Hospital Sydney Street London SW3 6NP UK E-mail: n.simmonds@imperial.ac.uk Received: Jan 052010 Accepted after revision: March 272010 First published online: April 082010 
(RBH; London, UK) and confirmed to have CF have been entered onto a database. The diagnosis is based on clinical features and a positive sweat sodium $\left(>70 \mathrm{mmol} \cdot \mathrm{L}^{-1}\right)$ or chloride $\left(>60 \mathrm{mmol} \cdot \mathrm{L}^{-1}\right)$ test or, in cases with a borderline or negative sweat test result, the presence of a known diseasecausing mutation on each CFTR gene, or of an abnormal nasal potential difference measurement. Patients were referred as adults from an adult physician or by their general practitioner, or directly through transition from paediatric clinics (at $\sim 15$ yrs of age). Clinical and demographic details are collected at the first consultation and are subsequently updated at annual review.

We studied only patients with a diagnosis of CF before the age of $17 \mathrm{yrs}$. These were identified from the database and classified as cases or controls as follows. Cases (long-term survivors) were all patients with complete records who had reached 40 yrs of age without transplantation by December 31, 2004. Controls were selected from all patients with complete records who had died before $30 \mathrm{yrs}$ of age or required transplantation at $<30$ yrs of age by December 31, 2004. We excluded controls $(n=27)$ who had died from a non-CF related cause (e.g. road traffic accident).

80 cases and 400 controls were identified from the original population. To ensure that cases and controls were similar in terms of era of birth, as it is likely that this would have influenced the nature of care received, cases were matched by date of birth ( \pm 365 days) to all eligible controls. Of the 80 cases identified, 78 were matched to at least one control. Each control was matched with as many cases as eligible and controls could be matched to more than one case. Of the 400 controls identified, 152 were matched to at least one case.

Information on source of referral, guardian's occupation, genotype and clinical state (weight, height, lung function, sputum microbiology, diabetic status, use of pancreatic enzymes, previous pneumothoraces, episodes of major haemoptysis and number of previous hospital admissions or antibiotic courses) prior to and at referral was collected from the initial assessment at the adult clinic; the remaining data were collected from annual reviews (school disruption, number of Advanced (" $\mathrm{A}^{\prime \prime}$ )-level school examinations and number of siblings). Antibiotic treatments before first attendance at the adult clinic were categorised as oral, aerosolised or i.v.

\section{Statistical analysis}

Differences between cases and controls were described by frequencies and proportions for categorical variables, and medians and interquartile ranges for continuous variables. Development of CF-related diabetes (CFRD) and the acquisition of Staphylococcus aureus, Pseudomonas aeruginosa and Haemophilus influenzae were assessed in terms of whether the patient developed these conditions before the age of 16 yrs. As such, analyses of these variables were limited to those who arrived at RBH by 16 yrs of age (69 cases and 109 controls). Physical measurements at initial assessment, history of antibiotic use and number of hospital admissions prior to initial assessment were limited to those arriving at $\mathrm{RBH}$ by the age of 15 yrs (73 cases and 131 controls).

We used probability-weighted logistic regression models to assess the association between possible predictors and survival to 40 yrs of age (case status). Using this method, controls were weighted according to the cases to which they were matched; thus, making the distribution of the matching variable (date of birth) similar in both groups. Each control was weighted by the sum, across its matched case, of $1 /$ (number of controls to which the case is matched). Cases were allocated a weight of 1 . Model results are presented as OR and $95 \%$ CI. Since patients were transferred to the adult clinic at varying ages, ORs for physical measures and medical history prior to initial assessment (use of antibiotics, prior hospital admissions, history of pneumothorax and major haemoptysis prior to initial assessment) were adjusted for age at assessment. ORs for physical measures were also adjusted for sex. Analyses were conducted in SAS v9.1 (SAS Institute, Cary, NC, USA) or STATA (StataCorp LP, College Station, TX, USA).

\section{Ethics}

All patients consented for their anonymised data to be included in the database for research purposes. The study was approved by the RBH Research Ethics Committee.

\section{RESULTS}

\section{Clinical characteristics}

Half of the participants were born between 1960 and 1965 and most $(80.4 \%)$ were diagnosed with CF before the age of 5 yrs (table 1). 70\% were first seen in the adult clinic before $21 \mathrm{yrs}$ of age. $97 \%$ had pancreatic insufficiency and there were similar proportions of males in cases (long-term survivors) and controls. Genotyping was only possible for patients surviving beyond 1989 (i.e. the year CFTR was discovered); therefore, genetic data were available for 74 patients (67 cases). Of the long-term survivors genotyped $(86 \%), 32$ (48\%) were homozygous for $\Delta \mathrm{F} 508,13(19 \%)$ were compound heterozygous for $\Delta \mathrm{F} 508$ and $19(28 \%)$ were heterozygous for $\Delta \mathrm{F} 508$ (with an unidentifiable second CF mutation). The remaining three cases were $621+1 \mathrm{G} \rightarrow \mathrm{T}, \mathrm{R} 553 \mathrm{X}$ (both with unidentifiable second genes) and R347P/3659delC. The seven controls genotyped were homozygous $\Delta \mathrm{F} 508$.

Features significantly associated with case status (i.e. long-term survivors) included diagnosis after 5 yrs of age. Patients whose initial presentation had been with respiratory disease were significantly less likely to be cases. Patients who had suffered a pneumothorax prior to referral to the adult clinic were significantly less likely to be cases after adjusting for age at first attendance. There was little heterogeneity in the distributions of pancreatic insufficiency, haemoptysis and CFRD prior to referral; none was associated with case status. After adjusting for age at initial assessment and sex, the probability of survival to 40 yrs increased with increasing height, weight, body mass index (BMI), forced expiratory volume in $1 \mathrm{~s}$ (FEV1) and forced vital capacity as recorded at the initial assessment in the adult clinic.

\section{Sociodemographic factors and patients' educational background}

Associations between long-term survival and measures of socio-economic status and educational attainment are shown in table 2. Patients referred from paediatric clinic B (paediatric clinic in a low social economic status area) were less likely to be cases. Those whose guardians were in managerial or manual 
TABLE 1 Early characteristics and associations with case status (long-term survival)

\begin{tabular}{|c|c|c|c|c|c|}
\hline & All & Cases & Controls & OR $(95 \% \mathrm{Cl})$ & Wald test $p$-values \\
\hline Subjects $n$ & 230 & 78 & 152 & & \\
\hline Female & $103(44.8)$ & $34(43.4)$ & $69(45.4)$ & 1.00 & \\
\hline Male & $127(55.2)$ & $44(56.4)$ & $83(54.6)$ & $1.09(0.62-1.92)$ & 0.75 \\
\hline \multicolumn{6}{|l|}{ Original presenting features } \\
\hline Yes & $98(44.1)$ & $24(31.2)$ & $74(51.0)$ & $0.44(0.24-0.80)$ & 0.01 \\
\hline \multicolumn{6}{|c|}{ Malabsorption/failure to thrive } \\
\hline No & 78 (35.3) & $33(42.9)$ & $45(31.3)$ & 1.00 & \\
\hline Yes & $143(64.7)$ & $44(57.1)$ & $99(68.8)$ & $0.59(0.33-1.06)$ & 0.08 \\
\hline \multicolumn{6}{|l|}{ Relative with CF } \\
\hline No & $189(85.9)$ & $63(81.8)$ & $126(88.1)$ & 1.00 & \\
\hline Yes & $14(6.4)$ & $5(6.5)$ & $9(6.3)$ & $1.40(0.42-4.61)$ & 0.58 \\
\hline \multicolumn{6}{|l|}{ Other } \\
\hline No & $197(90.0)$ & $67(87.0)$ & $130(91.6)$ & 1.00 & \\
\hline Yes & $22(10.1)$ & $10(13.0)$ & $12(8.5)$ & $1.67(0.67-4.17)$ & 0.27 \\
\hline \multicolumn{6}{|l|}{ Age at diagnosis yrs } \\
\hline$<5$ & $185(80.4)$ & $55(70.5)$ & $130(85.5)$ & 1.00 & 0.02 \\
\hline$\geqslant 5$ & $45(19.6)$ & $23(29.5)$ & $22(14.5)$ & $2.31(1.17-4.56)$ & \\
\hline \multicolumn{6}{|c|}{ Prior to attendance at adult clinic } \\
\hline \multicolumn{6}{|l|}{ Use of pancreatic enzymes } \\
\hline No & $6(2.6)$ & $2(2.6)$ & $4(2.6)$ & 1.00 & 0.81 \\
\hline Yes & $224(97.4)$ & $76(97.4)$ & $148(97.4)$ & $0.81(0.14-4.64)$ & \\
\hline \multicolumn{6}{|l|}{ CFRD before age 16 yrs } \\
\hline Yes & $17(7.4)$ & $6(7.7)$ & $11(7.2)$ & $0.58(0.21-1.63)$ & \\
\hline \multicolumn{6}{|c|}{ At presentation to adult clinic } \\
\hline Height $\mathrm{cm}$ & $165(158-172)$ & $169(162-175)$ & $162(157.5-168.5)$ & $1.71(1.04-2.82)^{\natural}$ per $10 \mathrm{~cm}$ & 0.03 \\
\hline Weight kg & $49.5(43.8-56.2)$ & $55.5(48.8-62.4)$ & $45.8(39.6-50.3)$ & $2.25(1.51-3.34)^{*}$ per $5 \mathrm{~kg}$ & $<0.01$ \\
\hline Body mass index $\mathrm{kg} \cdot \mathrm{m}^{-2}$ & $17.8(16.5-19.9)$ & $19.6(17.8-21.5)$ & $16.8(15.7-18.2)$ & $1.76(1.40-2.22)^{\bullet}$ & $<0.01$ \\
\hline FEV $1 \%$ pred & $41.3(24.5-69.7)$ & $57.3(41.5-81.6)$ & $27.5(20.4-42.1)$ & $1.54(1.32-1.80)^{\circ}$ per $5 \%$ predicted value & $<0.01$ \\
\hline FVC \% pred & $61.3(40.3-81.4)$ & $75.9(60.1-94.8)$ & $43.2(32.3-66.3)$ & $1.54(1.33-1.78)^{\bullet}$ per $5 \%$ predicted value & $<0.01$ \\
\hline
\end{tabular}

Data are presented as $n(\%)$ or median (interquartile range), unless otherwise stated. ORs were derived from weighted logistic regression models. Analysis is limited to those attending from age $16 \mathrm{yrs}$. ORs were derived from weighted logistic regression models. CF: cystic fibrosis; CFRD: CF-related diabetes mellitus; FEV1: forced expiratory volume in $1 \mathrm{~s}$; \% pred: \% predicted; FVC: forced vital capacity. ${ }^{\#}$ : OR adjusted for age at initial assessment; ${ }^{\%}$ : OR adjusted for age at initial assessment and sex.

(skilled or unskilled) occupations were more likely to be cases than those in professional occupations, but the difference was not statistically significant. Patients classified as having "mildly" or "grossly" disrupted schooling were statistically more likely to be controls, but there was no association between case status and the number of A-levels achieved. We found no association between sibling number (with or without CF) and case status.

\section{Sputum microbiology, antibiotic courses and hospital admissions}

Table 3 displays the association between long-term survival and sputum microbiology, antibiotic courses and hospital admissions prior to referral to the adult clinic. Acquiring $P$. aeruginosa, but not $H$. influenzae or $S$. aureus, in the sputum prior to $16 \mathrm{yrs}$ of age, was associated with a reduced probability of being a case. 


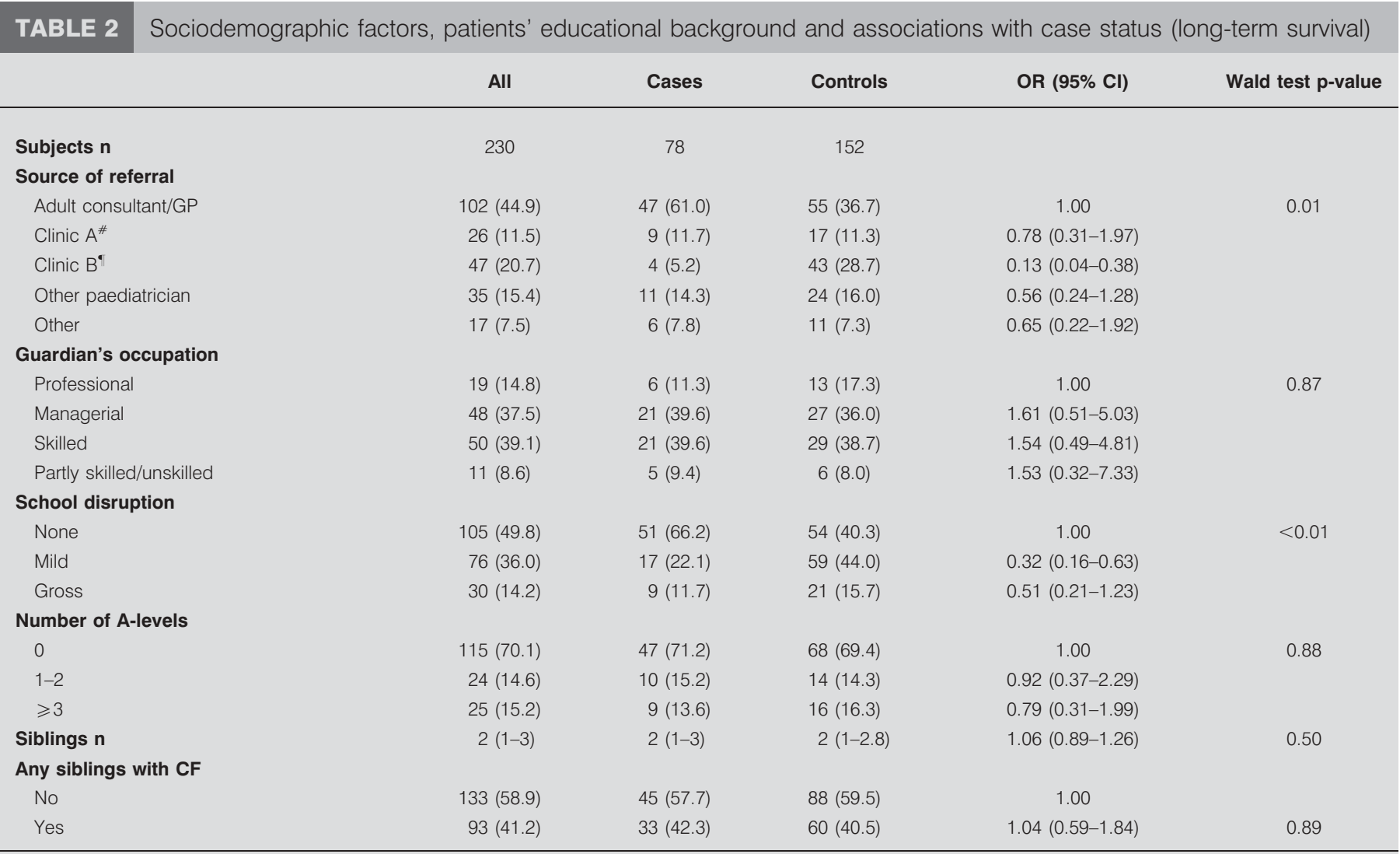

Data are presented as $n(\%)$ or median (interquartile range), unless otherwise stated. ORs were derived from weighted logistic regression models. GP: general practitioner; A-level: Advanced level school examination; CF; cystic fibrosis. ${ }^{\#}$ : clinic A is a paediatric clinic in a high socioeconomic status area; ${ }^{\circ}$ : clinic B is a paediatric clinic in a low socioeconomic status area.

Patients who had received oral antibiotics (as intermittent courses and/or long-term/prophylaxis), and had not received aerosolised or i.v. antibiotics, were significantly more likely to be cases than those who had not taken oral antibiotics. Conversely, the prior use of aerosolised or i.v. antibiotics was inversely associated with case status. Patients requiring annual or more frequent hospital admissions were significantly less likely to be cases.

\section{DISCUSSION}

This carefully matched case-control study is the first to report on the potential early influences of long-term survival in patients diagnosed with CF in childhood. Patients with a later diagnosis (i.e. at 5-16 yrs of age), those whose CF did not present with respiratory disease and those with higher weight, height, BMI and lung function (\% predicted) at the time of their first assessment at the adult clinic were statistically more likely to reach 40 yrs of age. Acquiring $P$. aeruginosa, but not $H$. influenzae or S. aureus, in the sputum prior to 16 yrs of age, was associated with a reduced probability of long-term survival. Factors that did not influence long-term survival included sex, parental occupation and major haemoptysis or the development of diabetes before 16 yrs of age. These findings suggest that the long-term survival of adults diagnosed with CF in childhood is determined predominantly by an intrinsically severe phenotype in early life, with little evidence of major modification by socioeconomic influences, and that maintaining good health in childhood is an important determinant of long-term survival.

We elected to study only patients whose disease had been diagnosed during childhood, and thus remove the bias associated with the good prognosis of disease when diagnosed in adulthood [10, 11]. Moreover, by studying long-term survivors under the care of a single institution and by matching them with "controls" born within a year of their birth date, we reduced the effects of different adult treatment strategies between centres and changing strategies over time, each of which may have independent effects on survival [9]. We may, in this way, have "over-matched" patients, leaving insufficient heterogeneity of exposure to examine some important determinants of survival. For example, it is widely accepted that socioeconomic factors have a strong influence on prognosis [9, 12, 13] but our findings demonstrated only limited evidence of this. In contrast to a previous UK study in 1989, we found no correlation of parental occupation (an index of family socioeconomic status) with long-term survival [14]. The association of poor survival with referral from paediatric clinic B (situated in an area of relatively low socioeconomic status) may reflect differences in resources and provision of care, as well as patients' sociodemographics. 
TABLE 3 Sputum microbiology, antibiotic treatment and hospital admissions prior to referral to the adult clinic and associations with case status (long-term survival)

\begin{tabular}{|c|c|c|c|c|c|}
\hline & All & Cases & Controls & OR $(95 \% \mathrm{Cl})$ & Wald test $p$-value \\
\hline Subjects $\mathbf{n}$ & 230 & 78 & 152 & & \\
\hline \multicolumn{6}{|l|}{ Microbiology $\#$} \\
\hline No & $148(84.6)$ & $61(91.0)$ & 87 (80.6) & 1.00 & 0.06 \\
\hline Yes & $27(15.4)$ & $6(9.0)$ & $21(19.4)$ & $0.40(0.15-1.06)$ & \\
\hline Yes & $25(14.2)$ & $3(4.4)$ & $22(20.4)$ & $0.18(0.05-0.65)$ & \\
\hline \multicolumn{6}{|l|}{ Haemophilus influenzae } \\
\hline No & $159(90.3)$ & $63(92.7)$ & $96(88.9)$ & 1.00 & 0.42 \\
\hline Yes & $17(9.7)$ & $5(7.4)$ & $12(11.1)$ & $0.63(0.21-1.92)$ & \\
\hline \multicolumn{6}{|l|}{ Oral antibiotics $^{+}$} \\
\hline No oral antibiotics & $54(28.6)$ & $8(11.4)$ & $46(38.7)$ & $1.00^{\S}$ & $<0.01$ \\
\hline Yes & $62(31.0)$ & $12(16.9)$ & $50(38.8)$ & $0.16(0.06-0.48)$ & \\
\hline \multicolumn{6}{|l|}{ Intravenous antibiotics ${ }^{+}$} \\
\hline No & $124(63.3)$ & $52(73.2)$ & $72(57.6)$ & $1.00^{\S}$ & $<0.01$ \\
\hline Yes & $72(36.7)$ & $19(26.8)$ & $53(42.4)$ & $0.15(0.05-0.42)$ & \\
\hline \multicolumn{6}{|c|}{ Previous hospital admissions $\mathrm{n}$} \\
\hline 0 & $16(8.3)$ & $13(18.8)$ & $3(2.4)$ & $1.00^{\S}$ & 0.01 \\
\hline $1-5$ & $178(91.8)$ & $56(81.2)$ & $122(97.6)$ & $0.17(0.04-0.70)$ & \\
\hline
\end{tabular}

Data are presented as $n(\%)$, unless otherwise stated. ORs were derived from weighted logistic regression models. " : acquisition before 16 yrs of age; ": analysis was limited to those attending from 16 yrs of age; ${ }^{+}$: analysis was limited to those attending from $15 \mathrm{yrs}$ of age; ${ }^{\text {s: }}$ OR adjusted for age at initial assessment.

However, the present study provides an important extra dimension to published studies on predictors of mortality. The earliest, observational, studies recognised the association of poor nutritional status and low FEV1 with a worse outcome [15-17]. Since then, more robust epidemiological studies have confirmed this correlation, including a large population study of the Canadian Patient Data Registry [3]. More recently, an Irish study investigated factors relating to mortality in their adult patients, concluding that lower FEV1 and BMI, and higher infection rates of $P$. aeruginosa and Burkholderia cepacia were associated with patients who had died [18]. They assessed differences in predetermined clinical parameters between patients who died during a 10-yr period and those who remained alive, therefore making it difficult to draw conclusions about the timing of the events (i.e. when they were most influential). Our study adds to this by clearly showing the importance of these factors at an early stage.

The present study demonstrated a worse outcome in patients diagnosed with CF early (before 5 yrs of age) and also in those with an initial disease presentation of respiratory symptoms. This supports the findings of a US registry-based study, demonstrating variable survival among patients with inherently different degrees of baseline risk, reflected by their age at diagnosis and their degree of disease severity at presentation [19]. They also showed that meconium ileus was associated with reduced survival, which provides an explanation for the lack of correlation found in our study, as only a few patients presenting with meconium ileus survived to adulthood. Contrary to their findings, we found that sex did not predict survival, which, in part, might be explained by the historical higher mortality among CF females, particularly around puberty, taking its toll, thus leaving those who have a predetermined survival advantage to progress through to the adult clinic [20]. However, others have argued that the socalled "gender gap" does not exist, highlighting the complex interaction of this much-debated relationship [21]. Patients with an increased baseline risk are predisposed to developing worse lung disease and an accelerated decline in their general health. Consequently, they develop more complications and ultimately require more hospital admissions and i.v. antibiotic courses, as demonstrated by the strong correlation of these factors with control status in our study.

The negative impact on survival of $P$. aeruginosa infection is consistent with previous studies and, although there is still some controversy regarding causality and ascertainment bias, it should be regarded as a poor prognostic factor $[22,23]$. The insignificant impact of $H$. influenzae and $S$. aureus is consistent with other studies. A European cross-sectional study demonstrated that $S$. aureus was not associated with worse pulmonary status and others have shown a deleterious effect on symptoms only, including the risk of massive haemoptysis [24-26]. The finding of a survival benefit for patients receiving oral 
antibiotics (without aerosolised or i.v. antibiotics) is interesting, as oral flucloxicillin is usually given as long-term prophylactic anti-staphylococcal treatment, suggesting indirectly that $S$. aureus may be relevant to survival, although this association may also be an indicator of milder disease [27].

We were unable to explore the impact on survival of specific CFTR mutations, as the majority of controls died before the discovery of the CF gene in 1989, making regression analysis impossible [28]. However, as $48 \%$ of the long-term survivors were homozygous for $\Delta \mathrm{F} 508$ (compared with $50 \%$ in the total UK adult CF population [29]), their survival advantage cannot be attributed to "milder" genotypes with less severe disease expression. We chose to use 17 yrs of age as our age criterion, as it has been demonstrated previously that this differentiates two distinct phenotypes of long-term survivors [11]. We acknowledge that we cannot be certain that all non-classic phenotypes have been excluded but combined with the genotype data and the fact that $97 \%$ of the total study population had pancreatic insufficiency, bias from genuine non-classic disease would have been minimal. Additionally, the use of a younger age of diagnosis would have further selected out "mild" cases; but with the recognition of significant disease heterogeneity even for homozygous $\Delta \mathrm{F} 508$, reducing the age would have excluded patients with "classic" disease genotypes that follow a milder disease course (e.g. due to gene modifiers), i.e. the group of patients of particular interest to this study.

There are several limitations to our findings. The incidence of complications such as CFRD and major haemoptysis increase with age [24], thus numbers were small in both groups at the time of assessment in the adult clinic, limiting the likelihood of finding an effect on survival. We were unable to assess the impact of B. cepacia complex infection as the importance of this pathogen in CF became apparent only in the mid-1980s [30]. Asymptomatic patients, diagnosed at birth through neonatal screening, are also not included in this study, as such programmes have only recently been introduced. The study was further limited by the data available to us and, therefore, in some instances, proxy markers (e.g. parental occupation) had to be used and patient numbers were small, making interpretation difficult. The information on socioeconomic status was therefore limited, as the broad category of "parental occupation" and the recognised limitations of "source of referral" do not allow for definitive conclusions to be made.

In summary, this study demonstrates the importance for longterm survival of achieving optimal growth and lung health by the time a patient attends an adult clinic. Effective clinical care is needed to facilitate this but, from our findings, we conclude that longevity is determined early, possibly by factors independent of CFTR function (e.g. gene modifiers) that determine early phenotype, disease severity and, ultimately, the probability of long-term survival.

\section{STATEMENT OF INTEREST}

None declared.

\section{REFERENCES}

1 Dodge JA, Lewis PA, Stanton M, et al. Cystic fibrosis mortality and survival in the UK: 1947-2003. Eur Respir J 2007; 29: 522-526.
2 Mahadeva R, Webb K, Westerbeek RC, et al. Clinical outcome in relation to care in centres specialising in cystic fibrosis: cross sectional study. BMJ 1998; 316: 1771-1775.

3 Corey M, Farewell V. Determinants of mortality from cystic fibrosis in Canada, 1970-1989. Am J Epidemiol 1996; 143: 1007-1017.

4 Jaffe A, Bush A. Cystic fibrosis: review of the decade. Monaldi Arch Chest Dis 2001; 56: 240-247.

5 De Boeck K, Wilschanski M, Castellani C, et al. Cystic fibrosis: terminology and diagnostic algorithms. Thorax 2006; 61: 627-635.

6 Simmonds NJ, Cullinan P, Hodson ME. Growing old with cystic fibrosis: the characteristics of long-term survivors of cystic fibrosis. Respir Med 2008; 103: 629-635.

7 Hodson ME, Simmonds NJ, Warwick WJ, et al. An international/ multicentre report on patients with cystic fibrosis (CF) over the age of 40 years. J Cyst Fibros 2008; 7: 537-542.

8 Drumm ML, Konstan MW, Schluchter MD, et al. Genetic modifiers of lung disease in cystic fibrosis. N Engl J Med 2005; 353: 1443-1453.

9 Schechter MS. Non-genetic influences on cystic fibrosis lung disease: the role of sociodemographic characteristics, environmental exposures, and healthcare interventions. Semin Respir Crit Care Med 2003; 24: 639-652.

10 Nick JA, Rodman DM. Manifestations of cystic fibrosis diagnosed in adulthood. Curr Opin Pulm Med 2005; 11: 513-518.

11 Rodman DM, Polis JM, Heltshe SL, et al. Late diagnosis defines a unique population of long-term survivors of cystic fibrosis. Am J Respir Crit Care Med 2005; 171: 621-626.

12 Schechter MS, Margolis PA. Relationship between socioeconomic status and disease severity in cystic fibrosis. J Pediatr 1998; 132: 260-264.

13 Schechter MS, Shelton BJ, Margolis PA, et al. The association of socioeconomic status with outcomes in cystic fibrosis patients in the United States. Am J Respir Crit Care Med 2001; 163: 1331-1337.

14 Britton JR. Effects of social class, sex, and region of residence on age at death from cystic fibrosis. BMJ 1989; 298: 483-487.

15 Kraemer R, Rudeberg A, Hadorn B, et al. Relative underweight in cystic fibrosis and its prognostic value. Acta Paediatr Scand 1978; 67: 33-37.

16 Corey M, McLaughlin FJ, Williams M, et al. A comparison of survival, growth, and pulmonary function in patients with cystic fibrosis in Boston and Toronto. J Clin Epidemiol 1988; 41: 583-591.

17 Huang NN, Schidlow DV, Szatrowski TH, et al. Clinical features, survival rate, and prognostic factors in young adults with cystic fibrosis. Am J Med 1987; 82: 871-879.

18 Courtney JM, Bradley J, Mccaughan J, et al. Predictors of mortality in adults with cystic fibrosis. Pediatr Pulmonol 2007; 42: 525-532.

19 Lai HJ, Cheng Y, Cho H, et al. Association between initial disease presentation, lung disease outcomes, and survival in patients with cystic fibrosis. Am J Epidemiol 2004; 159: 537-546.

20 Rosenfeld M, Davis R, FitzSimmons S, et al. Gender gap in cystic fibrosis mortality. Am J Epidemiol 1997; 145: 794-803.

21 Verma N, Bush A, Buchdahl R. Is there still a gender gap in cystic fibrosis? Chest 2005; 128: 2824-2834.

22 Emerson J, Rosenfeld M, McNamara S, et al. Pseudomonas aeruginosa and other predictors of mortality and morbidity in young children with cystic fibrosis. Pediatr Pulmonol 2002; 34: 91-100.

23 Henry RL, Mellis CM, Petrovic L. Mucoid Pseudomonas aeruginosa is a marker of poor survival in cystic fibrosis. Pediatr Pulmonol 1992; 12: 158-161.

24 Flume PA, Yankaskas JR, Ebeling M, et al. Massive hemoptysis in cystic fibrosis. Chest 2005; 128: 729-738.

25 Watkin SL, Elborn JS, Cordon SM, et al. C-reactive protein is not a useful indicator of intermittent bacterial colonization in early lung disease of patients with cystic fibrosis. Pediatr Pulmonol 1994; 17: 6-10.

26 Navarro J, Rainisio M, Harms HK, et al. Factors associated with poor pulmonary function: cross-sectional analysis of data from the 
ERCF. European Epidemiologic Registry of Cystic Fibrosis. Eur Respir J 2001; 18: 298-305.

27 Smyth A, Walters S. Prophylactic antibiotics for cystic fibrosis. Cochrane Database Syst Rev 2003; CD001912.

28 Kerem B, Rommens JM, Buchanan JA, et al. Identification of the cystic fibrosis gene: genetic analysis. Science 1989; 245: 1073-1080.
29 UK Cystic Fibrosis Database. Annual Data Report 2004. University of Dundee 2006. www.cystic-fibrosis.org.uk/pdfs/annualreport/ AuditReport2004.pdf Date last accessed: October 4, 2010. Date last updated: April, 2: 2010.

30 Isles A, Maclusky I, Corey M, et al. Pseudomonas cepacia infection in cystic fibrosis: an emerging problem. J Pediatr 1984; 104: 206-210. 